\title{
Severe Anemia Affects Both Splenectomized and Non- splenectomized Plasmodium falciparum-infected Aotus infulatus Monkeys
}

\author{
Leonardo J de Moura Carvalho/ ${ }^{+}$, Francisco Acácio Alves*/++, \\ Salma Gomes de Oliveira**, Rodrigo del Rio do Valle*, Andréa A Morais Fernandes/ ${ }^{+++}$, \\ José A Pereira Carneiro Muniz* ${ }^{*}$ Claudio T Daniel-Ribeiro/ ${ }^{++}$
}

\begin{abstract}
Laboratório de Pesquisas em Malária, Departamento de Imunologia, WHO-collaborating Centre for Research and Training in Immunology of Parasitic Diseases, Instituto Oswaldo Cruz-Fiocruz, Av. Brasil 4365, 21045-900 Rio de Janeiro, RJ, Brasil *Centro Nacional de Primatas **Instituto Evandro Chagas, Funasa, Belém, PA, Brasil
\end{abstract}

Severe anemia is the earliest and a frequently fatal complication of Plasmodium falciparum infection. Here we describe Aotus infulatus as a primate model suitable to study this malaria complication. Both non-splenectomized and splenectomized monkeys receiving different inocula of P. falciparum FVO strain presented large (> 50\%) decreases in hematocrit values during infection. Non-splenectomized animals were able to control parasite growth (parasitemia did not exceed 4\%), but they had to be treated because of severe anemia. Three of 4 splenectomized monkeys did not control parasitemia and were treated, but developed severe anemia after treatment when presenting a negative blood film. Destruction of parasitized red blood cells alone cannot account for the degree of anemia. Non-splenectomized monkeys repeatedly infected with homologous parasites became rapidly and progressively resistant to reinfection and to the development of severe anemia. The data presented here point to $\mathrm{A}$. infulatus as a suitable model for studying the pathogenesis of severe malarial infection.

Key words: malaria - Plasmodium falciparum - Aotus infulatus - primate model

New world monkeys of the genera Aotus and Saimiri are the primate models recommended by the World Health Organization for studies in malaria (WHO/OMS 1988). They are mainly used in pre-clinical trials of malaria vaccine candidates (Stowers \& Miller 2001, Herrera et al. 2002) but also in other types of studies, such as adaptation of Plasmodium falciparum and $P$. vivax strains (Collins 1994), drug evaluation (Berman et al. 1994, Wengelnik et al. 2002), polymorphism, and variation of parasite antigens (Scherf et al. 1998), pathology (Weller et al. 1992, Robert et al. 1996), and basic studies on their immune system (Nino-Vasquez et al. 2000, Daubenberger et al. 2001). A. lemurinus griseimembra, from Colombia, is referred as the most reliable model (Herrera et al. 2002) but, due to its scarce availability, other species such as $A$. nancymai, $A$. vociferans and $A$. lemurinus lemurinus have been frequently used (Collins 1994, Gramzinski et al. 1999). We have previously studied the susceptibility of $A$. infulatus, a species found in the southeastern Amazon and available at the Brazilian National Primate Center, to P. falciparum FVO strain (Carvalho et al. 2000) and have observed that splenectomized animals were not able to control parasite

${ }^{+}$Corresponding author. Fax: +55-21-2598-4611. E-mail: leojmc@ioc.fiocruz.br

${ }^{++}$Recipient of a fellowship from the Brazilian National Research Council (CNPq)

${ }^{+++}$Recipient of a fellowship from Capes

Received 13 December 2002

Accepted 26 June 2003 growth, developing high parasitemia requiring treatment. Non-splenectomized animals, on the contrary, could keep low parasitemia for longer periods but developed severe anemia. The development of this complication, which has been described also in other species of Aotus during infection with P. falciparum (Jones et al. 2002, Egan et al. 2002), makes A. infulatus a potential model not only for vaccine trials but also for studies of the pathogenesis of severe malarial anemia, which is the earliest and most frequent life-threatening complication of malaria in children (Gupta et al. 1999). The pathophysiological mechanisms behind this complication are poorly understood and there is evidence that both increased destruction and decreased production of erythrocytes are involved (Ekvall 2003). In both cases, no single factor can be identified as a major cause for the observed anemia, which is rather complex and multifactorial (Menendez et al. 2000). The availability of an animal model, well characterized in terms of course and outcome of infection, is of great importance to study this malarial complication, because studies in humans are constrained by several limitations. In our previous study, a single high inoculum was administered to a few monkeys, not allowing definitive conclusions on the pattern and outcome of infection in this species. Here we have analyzed the course of $P$. falciparum (FVO strain) primary and repeated infections in a larger number of intact as well as splenectomized $A$. infulatus monkeys using different inocula.

\section{MATERIALS AND METHODS}

Animals - Fourteen captive-bred adult male A. infulatus monkeys, born and maintained at the National Primate Center/Funasa in Belém, Brazil, and weighing 900-1 $250 \mathrm{~g}$ 
were used in the infection experiments. The description of their background has already been made (Pieczarka \& Nagamachi 1988, Carvalho et al. 2000). Four of the animals were splenectomized nearly one year before inoculation. The other 10 animals were kept intact (non-splenectomized).

Parasites and infections - The FVO strain of P. falciparum adapted to Aotus monkeys was used. The 10 non-splenectomized monkeys were divided in 2 groups of 5 animals each and inoculated intravenously with 50000 or 500000 parasitized red blood cells (pRBC) obtained from a donor Aotus monkey. The follow up of infection included a daily evaluation of parasitemia by thick and thin Giemsa-stained blood films, hematocrit evaluation every 3 days starting from day 8 of infection and daily measurement of body (rectal) temperature. An experienced veterinarian performed daily clinical examinations. Monkeys were treated with mefloquine $(15 \mathrm{mg} / \mathrm{kg})$ if parasitemia reached $5 \%$ or above, in agreement with the threshold used for other species of Aotus (Jones et al. 2001, Egan et al. 2002), or in case of a $>50 \%$ decrease of hematocrit in relation to pre-infection values, or still if the monkeys presented other manifestations of severe disease (prostration, anorexia), and being further followed up until complete recovery.

The non-splenectomized monkeys received a second infection 3 months after curing the first one, with a larger inoculum (1 $000000 \mathrm{pRBC})$ and the same type of follow up was performed. They also got a third and a fourth infection with $100000 \mathrm{pRBC}$ each time, 4 and 6 months after curing the second infection.

A separate experiment was conducted with 4 splenectomized monkeys, which received one inoculum of $50000 \mathrm{pRBC}$. The follow up was essentially the same as for non-splenectomized animals during primary infection, but the threshold of parasitemia for treatment was determined as $7.5 \%$ because we had previously shown that splenectomized $A$. infulatus monkeys can bear higher parasitemia (Carvalho et al. 2000).

Ethical considerations - All experimental protocols were submitted to and approved by the Fiocruz Ethical Committee on Animal Use (CEUA-Fiocruz protocol number P0047-00).

\section{RESULTS}

Non-splenectomized monkeys, primary infection - Fig. 1 shows the course of parasitemia in non-splenectomized monkeys inoculated with $50000 \mathrm{pRBC}$ (group 1: Fig. 1a) or 500000 pRBC (group 2: Fig. 1b). Both groups displayed a similar pattern of parasite growth and were also similar in regard to the final outcome of infection. By day 6, parasites were detected in all monkeys and parasitemia increased until days 12-17. The growth was not exponential and the curve of parasitemia in most animals rather formed a plateau. The mean day of peak parasitemia was 14.8 (range: 12-17) in group 1 and 16.2 (range: 16-17) in group 2. Highest recorded parasitemia was $3.6 \%$ in group 1 (mean: $2.2 \%$ ) and $4 \%$ in group 2 (mean: $2.4 \%$ ), but in only 4 animals ( 2 in each group) parasitemia went over $3 \%$. Three animals (2 in group 1) were able to keep it below $1 \%$ throughout the follow-up period. In none of the monkeys parasitemia reached the threshold foreseen for treatment (5\%) and, in 8 of them, parasitemia decreased after the peak without antimalarial treatment.

Despite none of the animals reached the threshold of parasitemia foreseen for treatment, 9 out of the 10 animals had large decreases in hematocrit, with $45-72 \%$ reductions in relation to pre-inoculation values (Fig. 2a, b). The exception was monkey AH-ADJ, whose minimum hematocrit value was $36 \%$ on day 23 post-inoculation. Monkeys were treated and, for most of them, hematocrit values stopped decreasing right after treatment and steadily returned to pre-infection levels within a few weeks.

Some particular cases might be stressed. In the group 1, 2 monkeys (AH-AHQ and AH-AIQ) had sharp decreases in hematocrit values on day 17 (Fig. 2a); monkey AH-AIQ died. In the group 2, 3 monkeys (AH-AFF, AH-AED and AH-AHE) were treated because of prostration; monkey AH-AFF died 2 days after treatment and monkey AHAHE had a sharp decrease in hematocrit after treatment but recovered.

Interesting observations were also made in the curve of temperature. Aotus monkeys present a high normal body temperature, usually above $39^{\circ} \mathrm{C}$. In group 1 , all monkeys presented a similar body temperature curve showing an increase starting from day 6 or 7 of infection and a high temperature plateau up to days 15-17 (Fig. 3a). Afterwards temperature decreased and fluctuated in the levels seen previous to days 6-7. The rise in temperature and the period of its maintenance closely fitted the curve of parasitemia. In group 2, there also seemed to be a rise in body temperature for all monkeys (Fig. 3b), but the picture was not so clear as for group 1 monkeys.

Splenectomized monkeys - Three of the 4 splenectomized naïve $A$. infulatus monkeys inoculated with $50000 \mathrm{pRBC}$ developed fast rising parasitemia reaching more than $10 \%$ and were treated on day 16 (Fig. 1c). Hematocrit values ranged from 32 to $36 \%$ at the time of treatment. However, contrary to what was observed with the non-splenectomized animals, hematocrit kept decreasing after treatment, when monkeys presented negative blood films, and reached values as low as 15\% 1 week after treatment (Fig. 2c). The fourth monkey (AHADD) was able to control parasite growth with peak parasitemia (1.6\%) being recorded on day 21 of infection and it was treated 3 days later with a hematocrit value down to $23 \%$. The 4 monkeys had a $>50 \%$ decrease in hematocrit in relation to pre-inoculation values, but they were able to recover with intensive care.

Non-splenectomized monkeys, repeated infections Three months after curing the first infection, the 8 remnant non-splenectomized monkeys were submitted to a second infection with $1000000 \mathrm{pRBC}$ of the same parasite and strain. Parasites were detected in all but 1 monkey (AHADJ) at low densities (Fig. 4). For most monkeys, parasitemia was kept below $0.1 \%$ and parasites were detected for only a few days. Highest peak parasitemia was $0.2 \%$ in monkey AH-AHQ, which also showed the longest lasting infection. All monkeys were able to control parasite growth, but 3 of the 8 (AH-ADM, AH-AED and AH-ADU) had decreases in hematocrit below 30\% (data not shown). The other monkeys self-cured the infection without relevant reduction in hematocrit. 

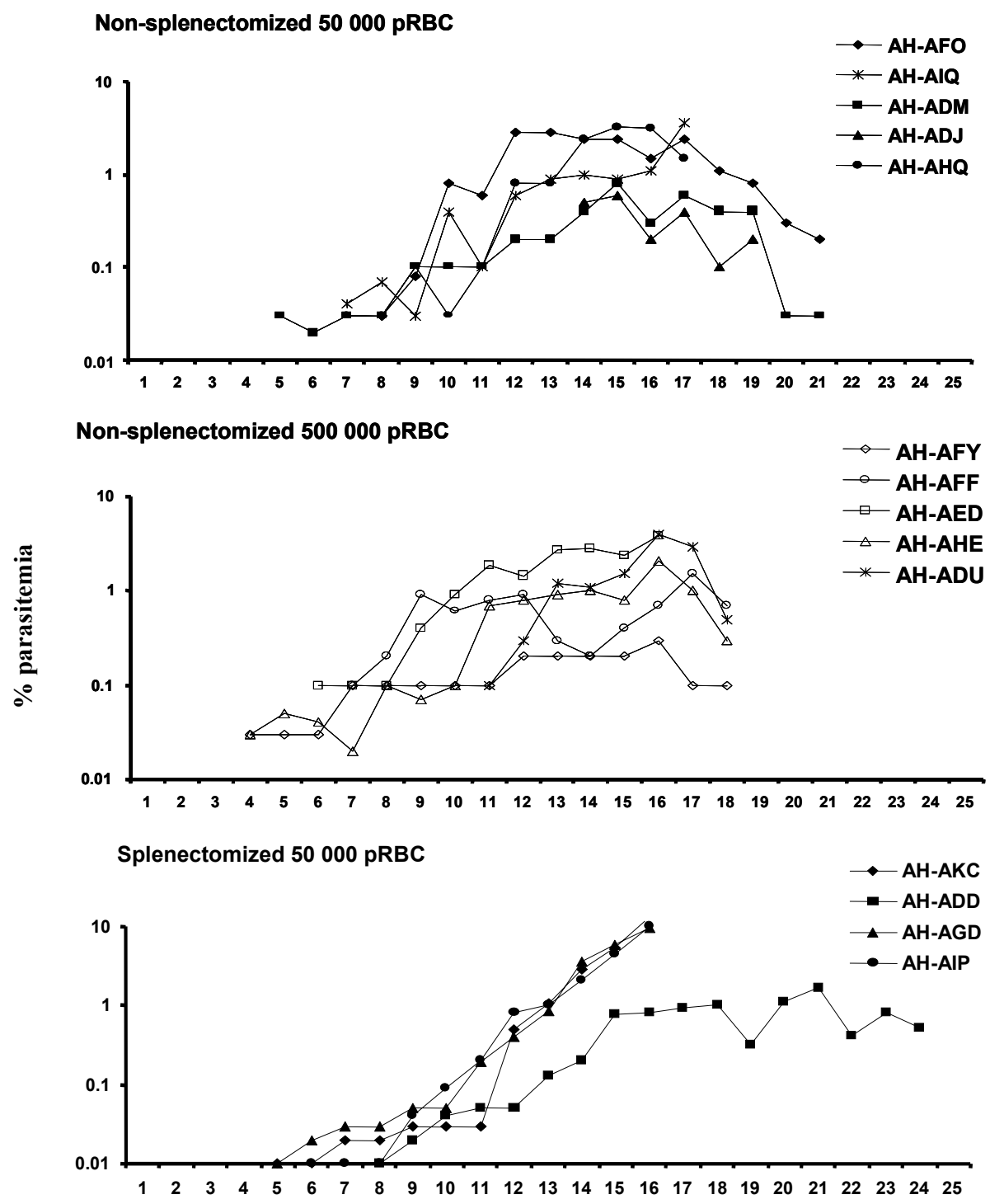

Days after inoculation

Fig. 1: course of parasitemia in naïve Aotus infulatus monkeys inoculated with Plasmodium falciparum FVO strain. The day of endpoint parasitemia is that of treatment, except for monkey AH-AIQ that died before being treated.

When submitted to a third inoculation with a 100000 pRBC 4 months after curing the second infection, only 1 of the monkeys (AH-ADM) presented a transient parasitemia reaching a maximum of $0.03 \%$ and self cured, and none presented relevant decrease in hematocrit. Following a fourth inoculation with $100000 \mathrm{pRBC}$, none of the monkeys presented detectable parasitemia or reduction in hematocrit.

\section{DISCUSSION}

One major problem to unravel the mechanisms behind the induction of severe malarial anemia is the fact that most studies are conducted in humans, which imposes obvious limitations to the approaches used. In addition, variables very difficult to control and inherent to human studies are commonly present, such as duration of plasmodial infection, degree of immunity, concomitant infections, genetic background, sex, age, epidemiological setting and others (Ekvall 2003). All these variables make studies in humans extremely complicated and many times inconclusive.

On the other hand, animal models also present limitations and many times findings in these models are not relevant to humans, especially when using phylogenetically distant host-parasite combinations, such as the mouse infected with murine Plasmodia. Primate models offer better possibilities for these studies and, in the case of malaria, the Aotus and Saimiri genera are considered the best choices because, among other characteristics, they can be infected with human Plasmodia such as $P$. falciparum and $P$. vivax (WHO/OMS 1988). Despite several species belonging to these primate genera have been 


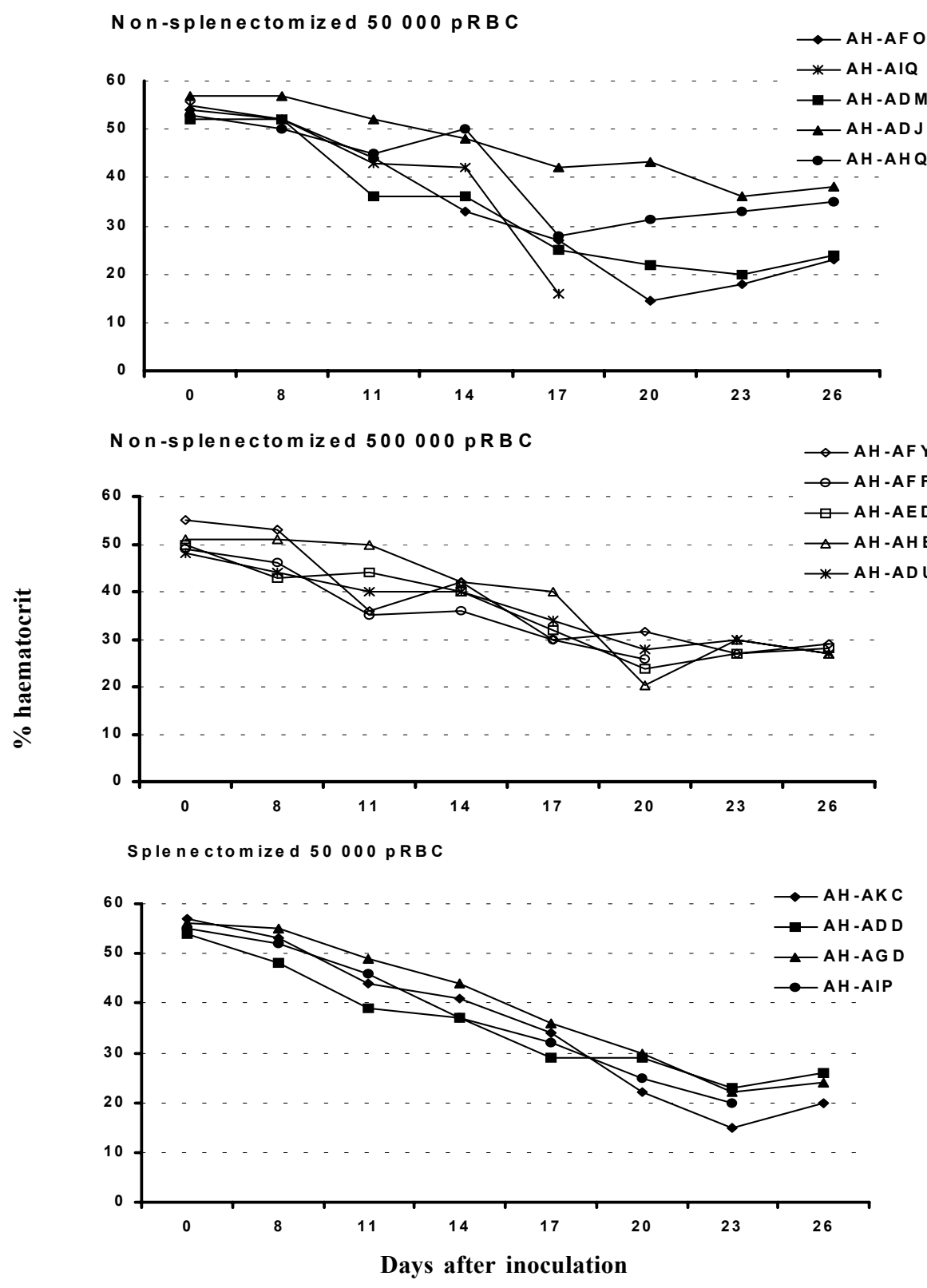

Fig. 2: hematocrit values during infection of naïve Aotus infulatus monkeys inoculated with Plasmodium falciparum FVO strain.

extensively used for malaria vaccine evaluation, little has been done in terms of developing a primate model for the study of malarial pathology, especially severe anemia. Following our report that $A$. infulatus could serve as such a model (Carvalho et al. 2000) 2 other works (Egan et al. 2002, Jones et al. 2002) have explored A. nancymai, $A$. vociferans and $A$. lemurinus lemurinus as potential models for severe malarial anemia, giving important contributions to this field. All these contributions open new roads for studying malarial anemia pathogenesis and helping unraveling the mechanisms behind this often fatal complication of $P$. falciparum infection. Here we add a new piece of work to expand our contribution to this field.

We have previously shown that both splenectomized and non-splenectomized $A$. infulatus monkeys were susceptible to infection by $P$. falciparum FVO strain and, as expected, splenectomized ones developed higher parasitemia whereas non-splenectomized monkeys presented lower parasitemia but developed severe anemia (Carvalho et al. 2000). Here we went further in the description of the characteristics of the infection in this novel primate model for malaria. The use of a larger number of animals allowed a better definition of the pattern of primary as well as repeated infections and the observation of new aspects. The most striking finding was the development of severe anemia in all but one animal irrespectively of the presence of the spleen or the level of parasitemia. The splenectomized animal followed up in the previous work did not develop severe anemia by day 18 (Carvalho et al. 2000), but since it was not further 


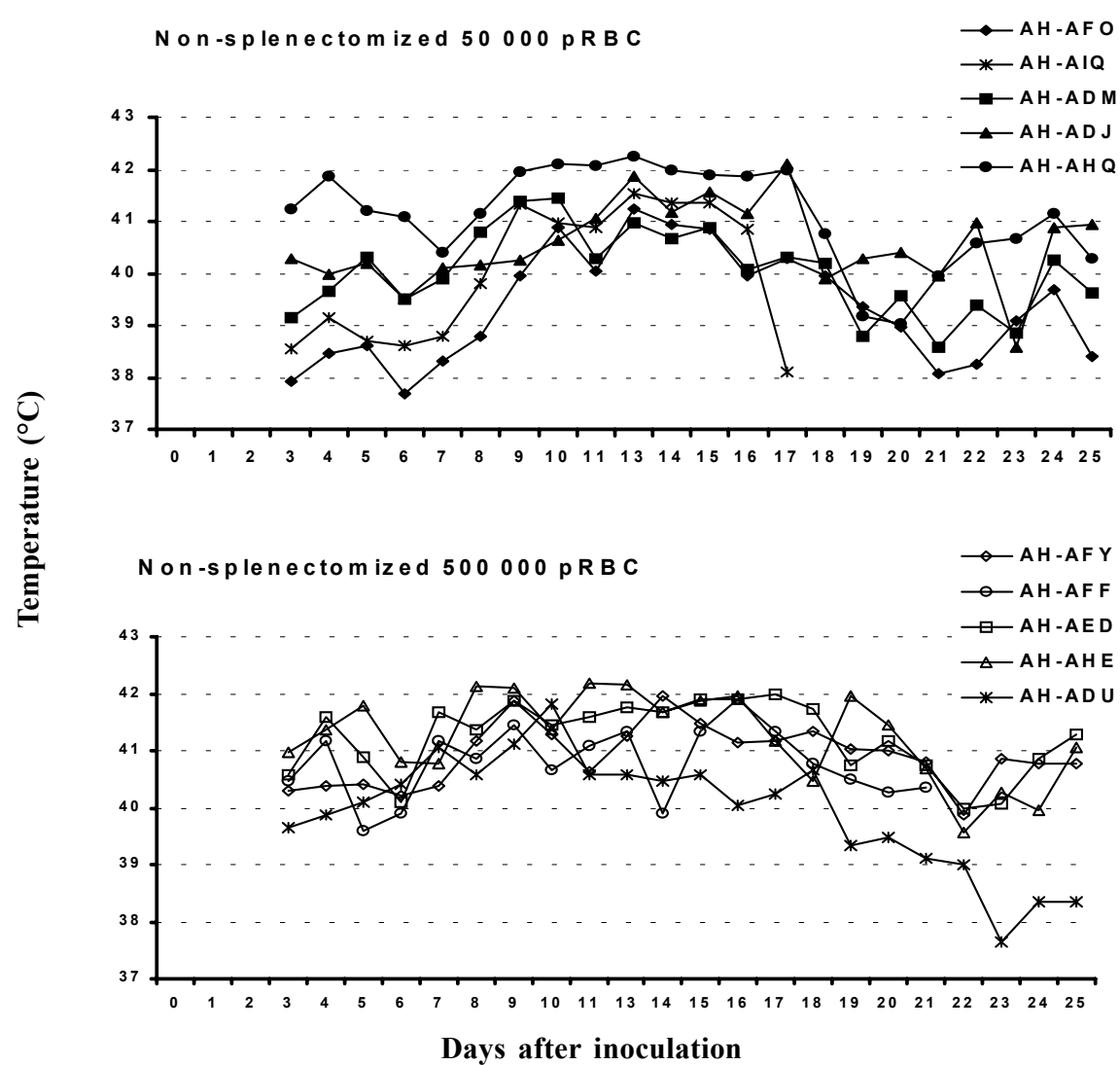

Fig. 3: curve of body temperature of non-splenectomized naïve Aotus infulatus monkeys inoculated with Plasmodium falciparum FVO strain.

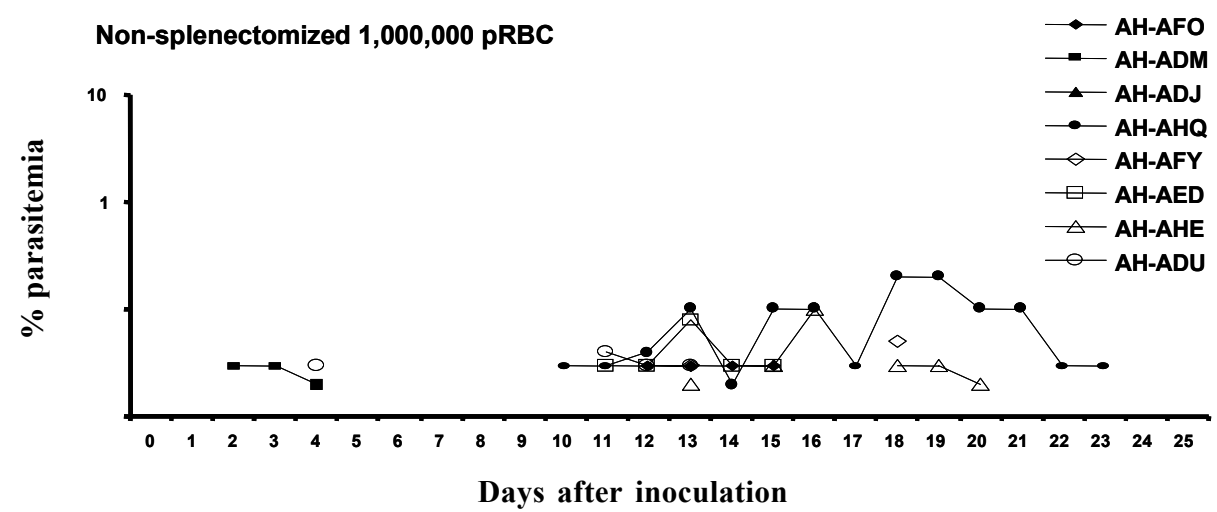

Fig. 4: course of parasitemia in non-splenectomized Aotus infulatus monkeys during a second infection with Plasmodium falciparum FVO strain. A high inoculum was given three months after monkeys had been treated of the primary infection. Only transient low parasitemia was detected in all animals.

followed up right after treatment this outcome cannot be ruled out. In fact, hematocrit values were similar in both studies, ranging from 29 to $36 \%$ at treatment and, in the present study, we have shown that hematocrit keep decreasing after treatment in the splenectomized animals.

Few other studies have directly addressed the question of malarial anemia in New World monkeys. Jones et al. (2002) showed that 12 of 50 non-splenectomized $A$. nancymai monkeys, immunized with EBA-175 DNA and recombinant vaccines or control formulations and challenged with $P$. falciparum FVO strain (primary infection), had reductions in hematocrit of $>50 \%$ in relation to pre-challenge values. Noteworthy is that, for many monkeys treated for elevated parasitemia, hematocrit continued to decrease after treatment. Three out of $8 \mathrm{~A}$. lemurinus lemurinus monkeys repeatedly infected with the P. falciparum $\mathrm{FVO}$ strain and then challenged with the heterologous CAMP strain also experienced 
reductions in hematocrit of $>50 \%$, while presenting low parasitemia. Egan et al. (2002) have described the occurrence of severe or moderate anemia in vaccinated A. nancymai or A. vociferans monkeys after a second infection, in most cases when monkeys presented low $(<5 \%)$ or even microscopically undetectable, PCRpositive, parasitemia.

In the present work, the frequency $(9 / 10)$ of severe malarial anemia in non-splenectomized $A$. infulatus monkeys was much higher than in the above-cited works. This might be due to a particular susceptibility of this species to malarial anemia, but other factors may have been important. One point is that monkeys in the present study were fully naive, i.e., they had experienced no previous malaria infections and had not been immunized with Plasmodium antigens. In what concerns previous malaria infections, it is difficult to make comparisons because, in the cited works, there was no reference to the outcome in terms of anemia in the primary infections in the immune or semi-immune monkeys (Egan et al. 2002). But the results with vaccinated animals showed that severe anemia induction was in fact not so frequent as in the present work. As stated, severe anemia was restricted to 12 of 50 animals when considering not only vaccinated but also control monkeys receiving nothing or control formulations (Jones et al. 2002). In addition, immunization did not seem to affect the induction of severe anemia, because it was not found an association between the priming with the immunogen and anemia.

We have used 2 inoculum sizes (50 000 and 500000 pRBC) in this work to check a possible interference of the initial parasite load on the course of parasitemia and development of anemia. However, the outcomes were very similar in the 2 groups of non-splenectomized animals, with variable parasitemias (peaks ranging from 0.3 to $4 \%$ ) reaching peak values on days 15-17 in 9 of the 10 monkeys. Therefore, a higher inoculum size was not predictive of higher or earlier peak parasitemia. It is possible that inoculum sizes lower than $50000 \mathrm{pRBC}$ or higher than 500000 pRBC lead to different outcomes. In any case, severe anemia occurred in 9 of the 10 animals, irrespectively of the parasitemia peak. So, whereas the initial parasite load studied has not allowed defining different outcomes, the results suggest that the parasite load during the course of infection was not critical for the development of anemia. This is reinforced by the fact that the 4 splenectomized animals also developed severe anemia, despite having variable peak parasitemias (ranging from $1.6 \%$ to $10 \%$ ) and different duration of infection (15 to 24 days). In humans, despite parasitemia seems to be higher in patients with severe anemia than in controls without this complication, the parasite load is not the critical point for anemia induction. In fact, destruction of $\mathrm{pRBC}$ alone cannot account for the degree of anemia in humans (Menendez et al. 2000) and in monkeys (Jones et al. 2002). This is also true here, since peak parasitemias as low as $0.3 \%$ produced the destruction of more than $50 \%$ of the RBC.

A fact of major importance is that non-splenectomized A. infulatus monkeys, contrary to A. nancymai and $A$. vociferans, were able to control parasite growth during a primary infection. As previously discussed by us (Carvalho et al. 2000) and Jones et al. (2002), partial control of parasitemia and its continuing low level must be more deleterious in relation to severe anemia than an exponential growth leading to high parasitemia. Early treatment due to high parasitemia might impair the appearance of conditions necessary for the induction of severe anemia. In this case, A. infulatus would not be particularly prone to malarial anemia, but would develop it because of bearing longer-lasting lower parasitemia. This is the case of Aotus immunized with partially protective malaria vaccines (Jones et al. 2001, Egan et al. 2002), and it seems also to be the case of P. falciparum-infected Saimiri monkeys (unpublished data). Development of severe anemia in Saimiri is not common, or at least not often reported, but in our experiments severe anemia also affected splenectomized Saimiri monkeys when parasitemia was kept for long in some animals partially protected with immunogens derived from the P. falciparum merozoite surface protein-3 (MSP3) (unpublished data). However, in the case of $A$. infulatus, this concept is challenged by the fact that also splenectomized monkeys developed severe anemia despite the early treatment due to high parasitemia. Overall, the data presented here suggest that A. infulatus is in fact particularly prone to this malaria complication.

In humans, severe anemia is the earliest and most frequent life-threatening complication of malaria in children living in areas of high transmission (Gupta et al. 1999). As stated, destruction of $\mathrm{pRBC}$ alone cannot account for the degree of anemia and there is some evidence that it may result from increased RBC (parasitized and non-parasitized) destruction as well as decreased RBC production through decreased erythroid proliferation or dyserythropoiesis (Ekvall 2003). Immune mechanisms seem to play a central role in both arms leading, for instance, to cytokine-induced dyserythropoiesis and non-parasitized RBC destruction or opsonization mediated by autoantibodies and spleen macrophages (see Daniel-Ribeiro \& Ferreira-da-Cruz 2001, and Weatherall et al. 2002 for review). Jones et al. (2002) have addressed the participation of antibodies and complement as possible mediators of non-parasitized RBC destruction, but failure in finding such mediators in RBC surface of infected monkeys suggested that this was not occurring. They also showed that bone marrow suppression occurs during infection. A central role is attributed to the spleen in the induction of malarial anemia in humans (Ekvall 2003). However, most data rely on indirect evidence and inferences. For instance, because the spleen is incriminated in the destruction of damaged or senescent RBC, because spleen macrophages seem to play important role in RBC destruction through phagocytosis, release of cytokines and reactive oxygen intermediates, and because spleen is enlarged during malaria infections (Menendez et al. 2000). However, the observation that $P$. falciparum-infected $A$. infulatus develop severe anemia in the absence of the spleen poses doubts on the proposed crucial role of this organ in the pathogenesis of this complication. This makes A. infulatus a precious model to address this and other issues related to the pathogenesis of severe malarial anemia. 
When considering anemia, there is always the possibility that coinfection with other blood pathogens such as Haemobartonella may interfere. However, this hemotropic parasite frequently observed in Saimiri and other mammals causes only latent infections in nonsplenectomized monkeys and, in this condition, seems not to interfere with plasmodial infection (Contamin \& Michil 1999). In case of splenectomized monkeys, interference seems to occur only when inoculation is given within 3-4 weeks following splenectomy, that is, when Haemobartonella infection becomes patent. So, it may be unlikely that anemia in A. infulatus in the present work had any major cause other than the plasmodial infection, because monkeys were either non-splenectomized or had been splenectomized one year before inoculation with $P$. falciparum.

Another finding was that $A$. infulatus monkeys become largely resistant to homologous $P$. falciparum challenge after only 1 infection, and apparently completely protected after 2 or 3 . They also become progressively resistant to anemia and, because humans seem to rapidly develop immunity against severe disease after 1 or 2 infections (Gupta et al. 1999), this may represent another similarity between $A$. infulatus and humans. The effect of heterologous challenge was not assessed in this work. Although direct comparison is not possible due to the large difference in the inocula and the intervals between infections, Jones et al. (2000) observed a similar pattern of acquisition of immunity using $A$. lemurinus lemurinus monkeys, although there was no reference concerning the hematocrit values after each challenge with the FVO strain. In that case, even using a low inoculum (10 000 pRBC), most monkeys were non-parasitemic when submitted to a fourth challenge infection, and those positive had very low parasitemia.

When considering A. infulatus as a model for malaria vaccine trials, 3 points must be discussed. First, assessing the capacity of vaccine candidate antigens in preventing severe anemia would be as important as their direct antiparasite effect. In fact, preventing complications especially in children must be a key goal of a malaria vaccine (Carvalho et al. 2002). Second, for vaccine trials, naïve animals shall preferentially be unable to spontaneously control parasite growth during a primary infection, so that they could be easily differentiated from those animals in which protection was eventually induced by vaccination. The higher resistance of non-splenectomized A. infulatus to the primary infection with $P$. falciparum $\mathrm{FVO}$ strain should represent a disadvantage in using this species for vaccine trials. However, this apparent limitation can be overcome with the enrollment of a proper number of animals per experimentation group, allowing statistical analysis of potential protective effects of test vaccines. Third, it is possible or likely that the dynamics of acquisition of antimalaria immunity in humans and in Aotus are different, but the extent of such a difference is difficult to assess. Under natural transmission, humans are inoculated with sporozoites rather than blood stage forms, and acquisition of immunity largely depends on the epidemiological setting (intensity of transmission). In addition, humans are exposed to a large variety of polymorphic plasmodial strains. It is known by ancient works of malariotherapy (Ciuca et al. 1924) that humans became immune with few inoculations with the same $P$. falciparum isolate. On the other hand, data obtained in Saimiri show that these monkeys, which usually acquire strong immunity after 1 or 2 inoculations with a homologous strain, may remain susceptible to heterologous challenge (Fandeur \& Chalvet 1998). In the present work, all challenges were carried out with the same strain (FVO). So, it is difficult to evaluate whether differences in the acquisition of immunity are due to real differences between humans and Aotus or to the experimental design. In any case, although primate models are not expected to be perfect counterparts of humans, they still represent unique resources for studying malaria pathogenesis and vaccines.

In conclusion, $A$. infulatus is a model that can be used in different types of studies related to malaria, especially those addressing severe malarial anemia. Its use as a model for vaccine trials is also indicated and further studies are necessary to determine its suitability for this purpose.

\section{ACKNOWLEDGEMENTS}

To Miguel Alfredo Sá da Costa for laboratory work and Maria de Fátima Ferreira da Cruz for critically reviewing this manuscript.

\section{REFERENCES}

Berman J, Brown L, Miller R, Andersen SL, McGreevy P, Schuster BG, Ellis W, Ager A, Rossan R 1994. Antimalarial activity of WR243251, a dihydroacridinedione. Antimicrob Agents Chemother 38: 1753-1756.

Carvalho LJM, Daniel-Ribeiro CT, Goto H 2002. Malaria vaccine: candidate antigens, mechanisms, constraints and prospects. Scand J Immunol 56: 327-343.

Carvalho LJM, Oliveira SG, Alves FA, Brígido MCO, Muniz JAPC, Daniel-Ribeiro CT 2000. Aotus infulatus is susceptible to Plasmodium falciparum infection and may constitute an alternative experimental model for malaria. Mem Inst Oswaldo Cruz 95: 363-365.

Ciuca M, Ballif L, Chelarescu-Virru M 1924. Immunity in malaria. Trans R Soc Trop Med Hyg 27: 619-622.

Collins WE 1994. The owl monkey as a model for malaria. In JR Baer, RE Weller, I Kakoma, (eds), Aotus: the Owl Monkey, Academic Press, New York, p. 254-258.

Contamin H, Michel JC 1999. Haemobartonellosis in squirrel monkeys (Saimiri sciureus): antagonism between Haemobartonella sp. and experimental Plasmodium falciparum malaria. Exp Parasitol 91: 297-305.

Daniel-Ribeiro CT, Ferreira-da-Cruz MF 2001. The new and the old in malaria immunopathology. Ci Cul 52: 269-281.

Daubenberger CA, Salomon M, Vecino W, Hubner B, Troll H, Rodrigues R, Patarroyo ME, Pluschke G 2001. Functional and structural similarity $\mathrm{V}$ gamma $9 \mathrm{~V}$ delta $2 \mathrm{~T}$ cells in humans and Aotus monkeys, a primate infection model for Plasmodium falciparum malaria. J Immunol 167: 64216430 .

Egan AF, Fabucci ME, Saul A, Kaslow D, Miller LH 2002. Aotus New World monkeys: model for studying malariainduced anemia. Blood 99: 3863-3866.

Ekvall H 2003. Malaria and anemia. Curr Opin Hematol 10: 108-114.

Gramzinski RA, Obaldia III N, Jones TR, Rossan RN, Collins WE, Garret DO, Lal AA, Hoffman SL 1999. Susceptibility of Panamanian Aotus lemurinus to sporozoite-induced 
Plasmodium falciparum (Santa Lucia) infection. Am J Trop Med Hyg 61: 19-25.

Fandeur T, Chalvet W 1998. Variant- and strain-specific immunity in Saimiri infected with Plasmodium falciparum. Am J Trop Med Hyg 58: 225-231.

Gupta S, Snow RW, Donnelly CA, Marsh K, Newbold C 1999. Immunity to non-cerebral severe malaria is acquired after one or two infections. Nat Med 5: 340-343.

Herrera S, Perlaza BL, Bonelo A, Arevalo-Herrera M 2002. Aotus monkeys: their great value for anti-malaria vaccines and drug testing. Int J Parasitol 32: 1625-1635.

Jones TR, Narum DL, Gozalo AS, Aguiar J, Fuhrmann SR, Liang H, Haynes JD, Moch JK, Lucas C, Luu T, Magill A, Hoffman SL, Sim BKL 2001. Protection of Aotus monkeys by Plasmodium falciparum EBA-175 region II DNA primeprotein boost immunization regimen. J Infect Dis 183: 303-312.

Jones TR, Obaldia III N, Gramzinski RA, Hoffman SL 2000. Repeated infection of Aotus monkeys with Plasmodium falciparum induces protection against subsequent challenge with homologous and heterologous strains of parasite. Am J Trop Med Hyg 62: 675-680.

Jones TR, Stroncek DF, Gozalo AS, Obaldia III N, Andersen EM, Lucas C, Narum DL, Magill AJ, Sim BKL, Hoffman SL 2002. Anemia in parasite- and recombinant proteinimmunized Aotus monkeys infected with Plasmodium falciparum. Am J Trop Med Hyg 66: 672-679.

Menendez C, Fleming AF, Alonso PL 2000. Malaria-related anemia. Parasitol Today 16: 469-476.

Nino-Vasquez JJ, Vogel D, Rodríguez R, Moreno A, Patarroyo ME, Pluschke G, Daubenberger CA 2000. Sequence and diversity of DRB genes of Aotus nancymai, a primate model for human malaria parasites. Immunogenetics 51:219-230.
Pieczarka JC, Nagamachi CY 1988. Cytogenetic studies of Aotus from eastern Amazonia: Y/autossome rearrangement. Am J Primatol 34: 197-204.

Robert C, Peyrol S, Pouvelle B, Gay-Andrieu F, Gysin J 1996. Ultrastructural aspects of Plasmodium falciparum-infected erythrocyte adherence to endothelial cells of Saimiri brain microvasculature. Am J Trop Med Hyg 54: 169-177.

Scherf A, Hernandez-Rivas R, Buffet P, Bottius E, Benatar C, Pouvelle B, Gysin J, Lanzer M 1998. Antigenic variation in malaria: in situ switching, relaxed and mutually exclusive transcription of var genes during intra-erythrocytic development in Plasmodium falciparum. EMBO J 17: 5418-5426.

Stowers A, Miller LH 2002. Are trials in New World monkeys on the critical path for blood-stage malaria vaccine development? Trends Parasitol 17: 415-419.

Weatherall DJ, Miller LH, Baruch DI, Marsh K, Doumbo OK, Casals-Pascual, Roberts DJ 2002. Malaria and the red cell. In VC Broudy, JL Abkowitz, JM Vose, JL Bajus, (eds), Hematology, American Society of Hematology Education Program Book, Washington DC, p. 35-57.

Weller RE, Collins WE, Buschbom RL, Ragan HA, Malaga CA 1992. Impaired renal function in owl monkeys (Aotus nancymai) infected with Plasmodium falciparum. Mem Inst Oswaldo Cruz 87(Suppl. 3): 435-42.

Wengelnik K, Vidal V, Ancelin ML, Cathiard AM, Morgat JL, Kocken CH, Calas M, Herrera S, Thomas AW, Vial HJ 2002. A class of potent antimalarials and their specific accumulation in infected erythrocytes. Science 295: 1311-1314.

WHO/OMS 1988. Memorandum from a WHO meeting: role of non-human primates in malaria vaccine development. Bull WHO/OMS 66: 719. 